\title{
Partial sharing of a set of meromorphic functions and normality
}

\author{
Virender Singh, Banarsi Lal, K.S. Charak and Shittal Sharma \\ Department of Mathematics, University of Jammu, Jammu, India \\ Received: 22 April 2016, Revised: 26 April 2016, Accepted: 28 April 2016 \\ Published online: 28 April 2016.
}

\begin{abstract}
By using the idea of partial sharing of a set of meromorphic functions by a member of a family of meromorphic functions and its $k$ th derivative we obtain a normality criterion generalizing some of the earlier results on shared sets and normal families of meromorphic functions. Further we prove a normality criterion which improves Marty's theorem and its reverse counterpart.
\end{abstract}

Keywords: Normal families, meromorphic function, partial sharing of sets, spherical derivative, nevanlinna theory.

\section{Introduction and Main Results}

Let $f$ be a nonconstant meromorphic function in the complex plane $\mathbb{C}$. We assume that the reader is familiar with the standard notions of the Nevanlinna value distribution theory such as $T(r, f), m(r, f), N(r, f)$ (see [6]). By $S(r, f)$, as usual, we shall mean a quantity that satisfies

$$
S(r, f)=\circ(T(r, f)) \text { as } r \rightarrow \infty
$$

possibly outside an exceptional set of finite logarithmic measure.

A family $\mathscr{F}$ of meromorphic functions defined on a domain $D \subseteq \overline{\mathbb{C}}$ is said to be normal in $D$ if every sequence of elements of $\mathscr{F}$ contains a subsequence which converges locally uniformly in $D$ with respect to the spherical metric, to a meromorphic function or $\infty$ (see [9]).

Two nonconstant meromorphic functions $f$ and $g$ defined on a domain $D$ are said to share a set $S$ of distinct meromorphic functions in $D$ if $\bigcup_{\phi \in S} \bar{E}_{f}(\phi)=\bigcup_{\phi \in S} \bar{E}_{g}(\phi)$, where $\bar{E}_{f}(\phi)=\{z \in D: f(z)=\phi(z)\}$. However, if $\bigcup_{\phi \in S} \bar{E}_{f}(\phi) \subseteq \bigcup_{\phi \in S} \bar{E}_{g}(\phi)$, then we say that $f$ share $S$ partially with $g$ and we write $f(z) \in S \Rightarrow g(z) \in S$.

Schwick [10] proved that if there exist three distinct finite value $a_{1}, a_{2}, a_{3}$ in $\mathbb{C}$ such that $f$ and $f^{\prime}$ share $a_{i}, i=1,2,3$ on $D$ for each $f \in \mathscr{F}$, then $\mathscr{F}$ is normal in $D$.

Fang [3] and Liu and Pang [7] extended the Schwick's result using the idea of shared sets. They precisely proved:

Theorem 1. Let $\mathscr{F}$ be a family of meromorphic functions in a domain $D$, and let $a_{1}, a_{2}$ and $a_{3}$ be three distinct finite complex numbers. If for every $f \in \mathscr{F}, f$ and $f^{\prime}$ share the set $S=\left\{a_{1}, a_{2}, a_{3}\right\}$, then $\mathscr{F}$ is normal in $D$.

In 2010, Chen [2] proved the following three results concerning a shared set of values: 
Theorem 2. Let $\mathscr{F}$ be a family of meromorphic functions in a domain $D$, and let $a_{1}, a_{2}$ and $a_{3}$ be three nonzero distinct finite complex numbers and let $S=\left\{a_{1}, a_{2}, a_{3}\right\}$. If for every $f \in \mathscr{F}, f(z) \in S \Rightarrow f^{\prime}(z) \in S$, then $\mathscr{F}$ is normal in $D$.

Theorem 3. Let $\mathscr{F}$ be a family of meromorphic functions in a domain D, all of whose poles are of multiplicity at least 3, let $a_{1}, a_{2}$ and $a_{3}$ be three distinct finite complex numbers, let $S=\left\{a_{1}, a_{2}, a_{3}\right\}$, and let $M$ be a positive number. If for every $f \in \mathscr{F},\left|f^{\prime}(z)\right| \leq M$ whenever $f(z) \in S$, then $\mathscr{F}$ is normal in $D$.

Theorem 4. Let $\mathscr{F}$ be a family of meromorphic functions in a domain D, all of whose zeros are multiple. Let $a_{1}$ and $a_{2}$ be two nonzero distinct finite complex numbers and let $S=\left\{a_{1}, a_{2}\right\}$. If for every $f \in \mathscr{F}, f(z) \in S \Rightarrow f^{\prime}(z) \in S$, then $\mathscr{F}$ is normal in $D$.

Chen [2] has given an example to show that the cardinality of $S$ in Theorem 2 and Theorem 3 cannot be reduced. But in Theorem 4, as far as we know, whether the condition on the multiplicity of the zeros and that on the values in $S$, are essential. We give here following examples to establish that these conditions are essential.

Example 1. Consider the family

$$
\mathscr{F}=\left\{f_{n}(z)=\tan n z: n=1,2, \cdots\right\}
$$

on the unit disk $\mathbb{D}$, and the set $S=\{i,-i\}$. Then each $f \in \mathscr{F}$ has simple zeros, and for every $f \in \mathscr{F}, f(z) \in S \Rightarrow f^{\prime}(z) \in S$. But $\mathscr{F}$ is not normal in $\mathbb{D}$. Thus the condition on the multiplicity of zeros is essential in Theorem 4.

Example 2. Consider the family

$$
\mathscr{F}=\left\{f_{n}(z)=\frac{e^{n z}}{n}: n=2,3, \cdots\right\}
$$

on the unit disk $\mathbb{D}$, and the set $S=\{0, \infty\}$. Then for every $f \in \mathscr{F}, f(z) \in S \Rightarrow f^{\prime}(z) \in S$. But $\mathscr{F}$ is not normal in $\mathbb{D}$. Thus the condition that $S$ has nonzero finite values is essential in Theorem 4.

In this paper, we generalize these results by replacing the elements of the shared set $S$ by distinct meromorphic functions as follows:

Let $\mathscr{F}$ be a family of meromorphic functions in a domain $D$, all of whose poles are of multiplicity at least 3 , and let $S:=\left\{\phi_{1}, \phi_{2}, \cdots, \phi_{n}\right\}$ be a set of $n$-distinct meromorphic functions in $D$, where $n \geq 3$.

\section{Theorem 5. If}

(i) for a given $m \in \mathbb{N}$ and for each $f \in \mathscr{F}, f(z) \in S \Rightarrow f^{(k)}(z) \in S, 1 \leq k \leq m$, and

(ii) $\forall z_{0} \in D$, the cardinality of the set $\left\{\phi_{1}\left(z_{0}\right), \phi_{2}\left(z_{0}\right), \cdots, \phi_{n}\left(z_{0}\right)\right\}$ is at most 2 implies that $f\left(z_{0}\right) \neq \phi_{i}\left(z_{0}\right)$ for at least 2 functions $\phi_{i}$ (depending on $f$ ),

then $\mathscr{F}$ is normal in $D$.

\section{Theorem 6. If}

(i) there is a constant $M>0$ such that $\left|f^{(k)}(z)\right| \leq M$ whenever $f(z) \in S \forall f \in \mathscr{F}, 1 \leq k \leq m$, where $m$ is a given positive integer, and

(ii) $\forall z_{0} \in D$, the cardinality of the set $\left\{\phi_{1}\left(z_{0}\right), \phi_{2}\left(z_{0}\right), \cdots, \phi_{n}\left(z_{0}\right)\right\}$ is at most 2 implies that $f\left(z_{0}\right) \neq \phi_{i}\left(z_{0}\right)$ for at least 2 functions $\phi_{i}$ (depending on $f$ ),

then $\mathscr{F}$ is normal in $D$.

Example 3. [4] Consider the family

$$
\mathscr{F}=\left\{f_{n}(z)=\frac{n+1}{2 n} e^{n z}+\frac{n-1}{2 n} e^{-n z}: n=2,3, \cdots\right\}
$$


on the unit disk $\mathbb{D}$ and set $S=\{-1,1\}$. Then for any $f_{n} \in \mathscr{F}$, we have $n^{2}\left[f_{n}^{2}(z)-1\right]=\left[f_{n}^{\prime}(z)\right]^{2}-1$. Thus $f_{n}(z) \in S \Rightarrow$ $f_{n}^{\prime}(z) \in S$ and $\left|f_{n}^{\prime}(z)\right| \leq 1$, but $\mathscr{F}$ is not normal in $\mathbb{D}$. This shows that the cardinality of $S$ in Theorem 5 and Theorem 6 cannot be reduced.

Example 4. Consider the family

$$
\mathscr{F}=\left\{f_{n}(z)=n z: n=1,2, \cdots\right\}
$$

on the unit disk $\mathbb{D}$, and set $S=\{0,-1, \infty\}$. Then $f_{n}(0) \in S$ but $f_{n}^{\prime}(0) \notin S$ and $\left|f_{n}^{\prime}(0)\right| \rightarrow \infty$ as $n \rightarrow \infty$. Note that $\mathscr{F}$ is not normal in $\mathbb{D}$. Thus condition $(i)$ in Theorem 5 and as well as in Theorem 6 is essential.

Example 5. Consider the family

$$
\mathscr{F}=\left\{f_{n}(z)=2 n z^{2}: n=1,2, \cdots\right\}
$$

on the unit disk $\mathbb{D}$. Let $S=\left\{\phi_{1}, \phi_{2}, \phi_{3}\right\}$, where $\phi_{1}(z)=z^{2}, \phi_{2}(z)=z^{2} / 2$ and $\phi_{3}(z)=z^{2} / 3$. Then for every $f \in \mathscr{F}, f(z) \in$ $S \Rightarrow f^{\prime}(z) \in S$ and $\left|f^{\prime}(z)\right| \leq M$, where $M$ is a positive number. However, the family $\mathscr{F}$ is not normal in $\mathbb{D}$. Note that $f_{n}(0)=\phi_{1}(0)=\phi_{2}(0)=\phi_{3}(0)$. Therefore, the condition $(i i)$ cannot be dropped in Theorem 5 and Theorem 6.

\section{Remark.}

1. If $m \geq 3$, then the conclusion of Theorem 5 and Theorem 6 hold without the condition on the multiplicity of the poles.

2. Since $\left|f^{\prime}(z)\right| \leq M$ implies $f^{\#}(z) \leq M$, Theorem 6 generalizes Marty's theorem by taking $m=1$.

Recently, Grahl and Nevo [5] gave the following reverse counterpart to Marty's theorem:

Theorem 7. Let some $M>0$ be given and set

$$
\mathscr{G}:=\left\{f \in \mathscr{M}(\mathbb{D}): f^{\#}(z) \geq M \text { for all } z \in \mathbb{D}\right\} .
$$

Then $\mathscr{G}$ is normal in $\mathbb{D}$.

Here, we prove a generalization of Theorem 7 as:

Theorem 8. Let $k$ and $n$ be two positive integers with $k \geq 2$ and $n \geq 3$. Let $\mathscr{H}$ be a family of meromorphic functions in a domain $D$, all of whose zeros are of multiplicity at least $k+1$, and let the set $S=\left\{\phi_{1}, \phi_{2}, \cdots, \phi_{n}\right\}$, where $\phi_{i}(i=1,2, \cdots, n)$ are meromorphic functions on $D$ such that $\phi_{i}(z) \neq \phi_{j}(z)$ for $i \neq j, z \in D$. If, for every $f \in \mathscr{H}$,

$$
f^{(k)}(z) \in S \Rightarrow f^{\#}(z) \geq M,
$$

where $M>0$ is a constant, then $\mathscr{H}$ is normal in $D$.

The following examples show that various conditions in Theorem 8 cannot be dropped:

Example 6. Consider the family

$$
\mathscr{H}=\left\{f_{n}(z)=\frac{1}{n z}: n=1,2, \cdots\right\}
$$

on the open unit disk $\mathbb{D}$, and let $S=\{0, \infty\}$. Clearly, for every $n, f_{n}^{(k)}(0) \in S \Rightarrow f_{n}^{\#}(0)=n \rightarrow \infty$ as $n \rightarrow \infty$. However, the family $\mathscr{H}$ is not normal in $\mathbb{D}$. Thus the cardinality of $S$ cannot be reduced.

Example 7. Consider the family

$$
\mathscr{H}=\left\{f_{n}(z)=n z^{k}: n=1,2, \cdots\right\}
$$

on the open unit disk $\mathbb{D}$, and let $S=\{0,1, \infty\}$. Clearly, for every $f \in \mathscr{H}, f^{(k)}(z) \in S \Rightarrow f^{\#}(z) \geq M$, for some positive constant $M$. However, the family $\mathscr{H}$ is not normal in $\mathbb{D}$. This shows that the condition on the multiplicity of zeros in Theorem 8 is essential. 
Example 8. Consider the family

$$
\mathscr{H}=\left\{f_{n}(z)=n z^{3}: n=1,2, \cdots\right\}
$$

on the open unit disk $\mathbb{D}$, and let $S=\{0,1, \infty\}$. Clearly, for every $f \in \mathscr{H}, f_{n}^{\prime \prime}(0) \in S \Rightarrow f^{\#}(0)=0$. However, the family $\mathscr{H}$ is not normal in $\mathbb{D}$. Therefore the condition " $f^{(k)}(z) \in S \Rightarrow f^{\#}(z) \geq M$ " is essential.

Throughout the paper, we shall denote the open disk with center at $z_{0}$ and radius $r$ by $D\left(z_{0}, r\right)$ and the punctured disk by $D^{*}\left(z_{0}, r\right)$.

\section{Proof of the main results}

We need the following results for the proof of our main results:

Lemma 1. [8] Let $\mathscr{F}$ be a family of functions meromorphic in $\mathbb{D}$ all of whose zeros have multiplicity at least $m$ and all of whose poles have multiplicity at least $p$. Then, if $\mathscr{F}$ is not normal at a point $z_{0} \in \mathbb{D}$, there exist, for each $\alpha:-p<\alpha<m$,

(i) a real number $r: r<1$,

(ii) points $z_{n}:\left|z_{n}\right|<r$,

(iii) positive numbers $\rho_{n}: \rho_{n} \rightarrow 0$,

(iv) functions $f_{n} \in F$ such that $g_{n}(\zeta)=\rho_{n}^{-\alpha} f_{n}\left(z_{n}+\rho_{n} \zeta\right)$ converges locally uniformly with respect to the spherical metric to $g(\zeta)$, where $g(\zeta)$ is a non constant meromorphic function on $\mathbb{C}$ and $g^{\#}(\zeta) \leq g^{\#}(0)=1$.

Lemma 2. [1] Let $\mathscr{F}$ be a family of meromorphic functions in a domain $\mathbb{D}$ and let a and $b$ be distinct functions holomorphic on $\mathbb{D}$. Suppose that, for any $f \in \mathscr{F}$ and any $z \in \mathbb{D}, f(z) \neq a(z)$ and $f(z) \neq b(z)$. If $\mathscr{F}$ is normal in $\mathbb{D}-\{0\}$, then $\mathscr{F}$ is normal in $\mathbb{D}$.

Proof. [Proof of Theorem 5.] Since normality is a local property, it is enough to show that $\mathscr{F}$ is normal at each $z_{0} \in D$. Let $S_{1}=\left\{\phi_{1}\left(z_{0}\right), \phi_{2}\left(z_{0}\right), \cdots, \phi_{n}\left(z_{0}\right)\right\}$. We distinguish the following cases:

Case 1. Suppose that all the values in $S_{1}$ are finite.

Here the following subcases arise:

Subcase 1.1. When cardinality of $S_{1}$ is at least three.

Suppose that $\mathscr{F}$ is not normal at $z_{0}$. Then by Lemma 1 , we can find a sequence $\left\{f_{j}\right\}$ in $\mathscr{F}$, a sequence $\left\{z_{j}\right\}$ of complex numbers with $z_{j} \rightarrow z_{0}$ and a sequence $\left\{\rho_{j}\right\}$ of positive real numbers with $\rho_{j} \rightarrow 0$ such that

$$
g_{j}(\zeta)=f_{j}\left(z_{j}+\rho_{j} \zeta\right)
$$

converges locally uniformly with respect to the spherical metric to a non-constant meromorphic function $g(\zeta)$ on $\mathbb{C}$, all of whose poles are of multiplicity at least 3 , such that $g^{\#}(\zeta) \leq g^{\#}(0)=1$ for all $\zeta \in \mathbb{C}$.

Clearly $g$ assume at least one of the values of $S_{1}$, otherwise $g$ becomes constant by Picard's theorem. Let $\zeta_{0} \in \mathbb{C}$ be such that $g\left(\zeta_{0}\right)-\phi_{i}\left(z_{0}\right)=0$, for some $i=1,2, \cdots, n$. Since $g(\zeta) \not \equiv \phi_{i}\left(z_{0}\right)$, by Hurwitz's theorem there exist a sequence of points $\zeta_{j} \rightarrow \zeta$ such that for sufficiently large $j$,

$$
g_{j}\left(\zeta_{j}\right)=f_{j}\left(z_{j}+\rho_{j} \zeta_{j}\right)=\phi_{i}\left(z_{j}+\rho_{j} \zeta_{j}\right) \in S
$$

By hypothesis, for every $f \in \mathscr{F}, f(z) \in S \Rightarrow f^{(k)}(z) \in S(k=1,2, \cdots, m)$, it follows that

$$
f_{j}^{(k)}\left(z_{j}+\rho_{j} \zeta_{j}\right) \in S
$$


and hence

$$
g_{j}^{(k)}\left(\zeta_{j}\right)=\rho_{j}^{k} f_{j}^{(k)}\left(z_{j}+\rho_{j} \zeta_{j}\right)=\rho_{j}^{k} \phi_{i}\left(z_{j}+\rho_{j} \zeta_{j}\right),
$$

for some $i=1,2, \cdots, n$ and $k=1,2, \cdots, m$. Therefore

$$
g^{(k)}\left(\zeta_{0}\right)=\lim _{j \rightarrow \infty} g_{j}^{(k)}\left(\zeta_{j}\right)=0
$$

for $k=1,2, \cdots, m$, and so $\zeta_{0}$ is a zero of multiplicity at least $m+1$ for $g(\zeta)-\phi_{i}\left(z_{0}\right),(i=1,2, \cdots, n)$.

Since poles of $g$ have multiplicity at least 3 , by Second fundamental theorem of Nevanlinna, we have

$$
\begin{aligned}
(n-1) T(r, g) & \leq \bar{N}\left(r, \frac{1}{g-\phi_{1}\left(z_{0}\right)}\right)+\bar{N}\left(r, \frac{1}{g-\phi_{2}\left(z_{0}\right)}\right)+\cdots+\bar{N}\left(r, \frac{1}{g-\phi_{n}\left(z_{0}\right)}\right)+\bar{N}(r, g)+S(r, g) \\
& \leq \frac{1}{m+1}\left[N\left(r, \frac{1}{g-\phi_{1}\left(z_{0}\right)}\right)+N\left(r, \frac{1}{g-\phi_{2}\left(z_{0}\right)}\right)+\cdots+N\left(r, \frac{1}{g-\phi_{n}\left(z_{0}\right)}\right)\right]+\frac{1}{3} N(r, g)+S(r, g) \\
& \leq \frac{n}{m+1} T(r, g)+\frac{1}{3} T(r, g)+S(r, g) \\
& =\frac{3 n+m+1}{3 m+3} T(r, g)+S(r, g)
\end{aligned}
$$

which is a contradiction as $n \geq 3$. Thus $\mathscr{F}$ is normal at $z_{0}$.

Subcase 1.2. When cardinality of $S_{1}$ is at most two.

By hypothesis (ii), $f\left(z_{0}\right) \neq \phi_{i}\left(z_{0}\right)$ for at least two functions $\phi_{i},(i=1,2, \cdots, n)$. So we can find a small neighbourhood, say $D\left(z_{0}, r\right)$ such that $\phi_{i}(z) \neq \phi_{j}(z)(1 \leq i, j \leq n)$ in $D^{*}\left(z_{0}, r\right)$. Thus by subcase $1.1, \mathscr{F}$ is normal in $D^{*}\left(z_{0}, r\right)$. Now we show that $\mathscr{F}$ is normal at $z_{0}$.

Since $f\left(z_{0}\right) \neq \phi_{i}\left(z_{0}\right)$ for at least two functions $\phi_{i}$ and each $\phi_{i}\left(z_{0}\right)$ is finite, we find that for every $f \in \mathscr{F}, f(z) \neq \phi_{i}(z)$ for at least two functions $\phi_{i}$ which are holomorphic in $D\left(z_{0}, r\right)$. Thus by Lemma $2, \mathscr{F}$ is normal at $z_{0}$.

Case 2. Suppose one of the value in $S_{1}$ is infinite.

Without loss of generality, assume that $\phi_{1}\left(z_{0}\right)=\infty$. We take $h \notin S_{1}$ and consider the family

$$
\mathscr{G}=\left\{g=\frac{1}{f-h}: f \in \mathscr{F}\right\} .
$$

Clearly for every $f \in \mathscr{F}$,

$$
f\left(z_{0}\right) \in S_{1} \text { implies } g\left(z_{0}\right) \in S_{2}=\left\{0, \frac{1}{\phi_{1}\left(z_{0}\right)-h}, \frac{1}{\phi_{2}\left(z_{0}\right)-h}, \cdots, \frac{1}{\phi_{n}\left(z_{0}\right)-h}\right\},
$$

with all the values in $S_{2}$ finite. So we can find a small neighbourhood $D\left(z_{0}, r\right)$ of $z_{0}$ such that

$$
f(z) \in S \text { implies } g(z) \in T=\left\{0, \frac{1}{\phi_{1}(z)-h}, \frac{1}{\phi_{2}(z)-h}, \cdots, \frac{1}{\phi_{n}(z)-h}\right\} .
$$

Thus by Case $1, \mathscr{G}$ is normal at $z_{0}$ and which in turn implies that the family $\mathscr{F}$ is normal at $z_{0}$. 
Proof. [Proof of Theorem 6.] Since normality is a local property, it is enough to show that $\mathscr{F}$ is normal at each $z_{0} \in D$. Let $S_{1}=\left\{\phi_{1}\left(z_{0}\right), \phi_{2}\left(z_{0}\right), \cdots, \phi_{n}\left(z_{0}\right)\right\}$.

The proof is similar to that of Theorem 5 except the case when all the values in $S_{1}$ are finite, and cardinality of $S_{1}$ is at least three. So here we consider that case only.

Suppose that $\mathscr{F}$ is not normal at $z_{0}$. Then by Lemma 1 , we can find a sequence $\left\{f_{j}\right\}$ in $\mathscr{F}$, a sequence $\left\{z_{j}\right\}$ of complex numbers with $z_{j} \rightarrow z_{0}$ and a sequence $\left\{\rho_{j}\right\}$ of positive real numbers with $\rho_{j} \rightarrow 0$ such that

$$
g_{j}(\zeta)=f_{j}\left(z_{j}+\rho_{j} \zeta\right)
$$

converges locally uniformly with respect to the spherical metric to a non-constant meromorphic function $g(\zeta)$ on $\mathbb{C}$, all of whose poles are of multiplicity at least 3 , such that $g^{\#}(\zeta) \leq g^{\#}(0)=1$ for all $\zeta \in \mathbb{C}$.

Clearly $g$ assume at least one of the value in set $S_{1}$, otherwise $g$ becomes constant by Picard's theorem. Let $\zeta_{0} \in \mathbb{C}$ be such that $g\left(\zeta_{0}\right)-\phi_{i}\left(z_{0}\right)=0$, for some $i=1,2, \cdots, n$. Since $g(\zeta) \not \equiv \phi_{i}\left(z_{0}\right)$, by Hurwitz's theorem there exist a sequence of points $\zeta_{j} \rightarrow \zeta$ such that for sufficiently large $j$,

$$
g_{j}\left(\zeta_{j}\right)=f_{j}\left(z_{j}+\rho_{j} \zeta_{j}\right)=\phi_{i}\left(z_{j}+\rho_{j} \zeta_{j}\right) \in S
$$

By hypothesis, for every $f \in \mathscr{F},\left|f^{(k)}(z)\right| \leq M$ whenever $f(z) \in S(k=1,2, \cdots, m)$, it follows that

$$
\left|f_{j}^{(k)}\left(z_{j}+\rho_{j} \zeta_{j}\right)\right| \leq M
$$

and hence

$$
\left|g_{j}^{(k)}\left(\zeta_{j}\right)\right|=\left|\rho_{j}^{k} f_{j}^{(k)}\left(z_{j}+\rho_{j} \zeta_{j}\right)\right| \leq \rho_{j}^{k} M
$$

for $k=1,2, \cdots, m$. Therefore

$$
g^{(k)}\left(\zeta_{0}\right)=\lim _{j \rightarrow \infty} g_{j}^{(k)}\left(\zeta_{j}\right)=0
$$

for $k=1,2, \cdots, m$, and so $\zeta_{0}$ is a zero of multiplicity at least $m+1$ for $g(\zeta)-\phi_{i}\left(z_{0}\right),(i=1,2, \cdots, n)$.

Since poles of $g$ have multiplicity at least 3, using the Second fundamental theorem of Nevanlinna we arrive at a contradiction (as obtained in the proof of Theorem 5) showing $\mathscr{F}$ is normal at $z_{0}$.

Proof. [Proof of Theorem 8.]Since normality is a local property, it is enough to show that $\mathscr{H}$ is normal at each $z_{0} \in D$. Suppose that $\mathscr{H}$ is not normal at some point $z_{0} \in D$. Then by Lemma 1 , we can find a sequence $\left\{f_{j}\right\}$ in $\mathscr{H}$, a sequence $\left\{z_{j}\right\}$ of complex numbers with $z_{j} \rightarrow z_{0}$ and a sequence $\left\{\rho_{j}\right\}$ of positive real numbers with $\rho_{j} \rightarrow 0$ such that

$$
g_{j}(\zeta)=\rho_{j}^{-k} f_{j}\left(z_{j}+\rho_{j} \zeta\right)
$$

converges locally uniformly with respect to the spherical metric to a non-constant meromorphic function $g(\zeta)$ on $\mathbb{C}$, all of whose zeros have multiplicity at least $k+1$, such that $g^{\#}(\zeta) \leq g^{\#}(0)=1$ for all $\zeta \in \mathbb{C}$.

Clearly $g^{(k)}$ assume at least one of the value $\phi_{i}\left(z_{0}\right),(i=1,2, \cdots, n)$, otherwise $g^{(k)}$ becomes constant by Picard's theorem. Let $\zeta_{0} \in \mathbb{C}$ be such that $g^{(k)}\left(\zeta_{0}\right)-\phi_{i}\left(z_{0}\right)=0$ for some $i=1,2, \cdots, n$. Clearly, $g^{(k)}(\zeta) \not \equiv \phi_{i}\left(z_{0}\right)$, for otherwise $g$ would be a polynomial of degree at most $k$, which is a contradiction. By Hurwitz theorem, there exist a sequence of 
points $\zeta_{j} \rightarrow \zeta_{0}$ such that for sufficiently large $j$, we have

$$
g_{j}^{(k)}\left(\zeta_{j}\right)=f_{j}^{(k)}\left(\zeta_{j}+\rho_{j} \zeta_{j}\right)=\phi_{i}\left(z_{j}+\rho_{j} \zeta_{j}\right) \in S
$$

By hypothesis, for every $f \in \mathscr{H}, f^{\#}(z) \geq M$ whenever $f^{(k)}(z) \in S$, it follows that

$$
f_{j}^{\#}\left(\zeta_{j}+\rho_{j} \zeta_{j}\right) \geq M
$$

and hence,

$$
\begin{aligned}
g^{\#}\left(\zeta_{0}\right) & =\lim _{j \rightarrow \infty} g_{j}^{\#}\left(\zeta_{j}\right) \\
& =\lim _{j \rightarrow \infty} \rho_{j}^{-k+1}\left(f_{j}\right)^{\#}\left(\zeta_{j}+\rho_{j} \zeta_{j}\right) \\
& \geq \lim _{j \rightarrow \infty} \rho_{j}^{-k+1} M \rightarrow \infty
\end{aligned}
$$

which is a contradiction to the fact that $g$ has bounded spherical derivative. Hence $\mathscr{H}$ is normal at $z_{0}$.

Acknowledgment. The work of first and second author is supported by CSIR India, and that of the fourth author is supported by the UGC India.

\section{References}

[1] Chang, J.M., Fang, M.L., Zalcman, L.Composite meromorphic functions and normal families. Proc. Roy. Soc. Edinburgh, Ser. A, 139, 57-72(2009).

[2] Chen, J.F.Shared sets and normal families of meromorphhic fuunctions. Rocky Mountain Journal of Mathematics (5) 40, 517-524 (2010).

[3] Fang, M.L.A note on sharing values and normality. Chinese J. Math. Study 29, 29-32 (1996).

[4] Fang, M.L., Zalcman, L.Normal families and uniqueness theorems for entire functions. J. Math. Anal. Appl. 280, $273-283$ (2003).

[5] Grahl J., Nevo, S.Spherical derivatives and normal families. J. Anal. Math., 117, 119-128 (2012).

[6] Hayman, W.K.Meromorphic Functions Oxford, UK: Clarendon Press. (1964).

[7] Liu, X.J., Pang, X.C.Shared values and normal families. Acta Math. Sinica. 50, 409-412 (2007).

[8] Pang, X., Zalcman, L.Normal families and shared values. Bull. Lond. Math. Soc. 32, 325-331 (2000).

[9] Schiff, J.L.Normal Families. Springer-Verlag, New York, 1993.

[10] Schwick, W.Sharing values and normality. Arch. Math. (Basel)59, 50-54 (1992). 